\title{
Comparison between Metastatic Spinal Tumour of Unknown Primary Tumour with a Known Primary
}

\author{
Wissam Jassim Sagban ${ }^{1}$, Samer Mohammed Saeed Ridha ${ }^{1}$, Zaki Noah Hasan ${ }^{2 *}$ \\ ${ }^{1}$ Neuroscience Teaching - Hospital, Baghdad, Iraq \\ ${ }^{2}$ Department of Medicine, Al-Kindy College of Medicine, University of Baghdad, Baghdad, Iraq \\ Email: zaki_nooh@yahoo.com
}

Received 29 November 2015; accepted 31 January 2016; published 3 February 2016

Copyright (C) 2016 by authors and Scientific Research Publishing Inc.

This work is licensed under the Creative Commons Attribution International License (CC BY). http://creativecommons.org/licenses/by/4.0/

(c) (i) Open Access

\section{Abstract}

Patients presented with spinal metastases from unknown primary tumours are rare. In this research we evaluated all patients with metastatic spinal tumour of unknown primary tumour, all patients were evaluated pre-operatively and comparison was done to those patients who had known primary tumour the study aimed to compare the group with known primary and the group with the unknown primary regarding the: mean age, gender, duration of symptoms, complication rate, region of the spine affected by metastasis, presence or absence of other skeletal or visceral metastasis, histological cell type and neurological outcome. Method: A retrospective study was performed on 40 patients presented to Neuroscience hospital in Baghdad from January 2010 till January 2014; all patients with metastatic spinal tumour were included in our study. We reviewed all patients' records including age, sex, primary tumour, duration of their symptoms, neurological out come and complications. Results: Out of the 40 patient who presented with spinal tumours that underwent surgery duo to metastatic spinal tumour, five patients presented with spinal compression duo to metastatic tumour of unknown primary tumour $(12.5 \%)$. The mean age was 64 years, 4 male and one female. For those with a known primary tumour mean age was 61 year, 22 male 13 female. Duration of symptoms prior to surgery was the same 180 days for those with unknown primary and 190 day for those with known primary tumours. They also had similar neurological outcome $(80 \%)$ remain the same or improved post operatively for those with unknown primary and (85.7\%) for those with a known primary tumour, and a similar complication rate for unknown primary was $25 \%$ versus $28 \%$ for known. The primary site of metastatic spinal tumour of unknown primary was confirmed after histopathology all shown adenocarcinoma four from the lung (80\%) and one from colorectal. While the most common known carcinoma site were the lung 18 patient (51.4\%), colorectal $8(22.8 \%)$, breast $7(20 \%)$, and renal $2(5.71 \%)$. All patient who complain from secondary spinal metastasis with unknown primary tumour didn't show any other

\footnotetext{
${ }^{*}$ Corresponding author.
} 
skeletal or visceral metastasis, while those with a known primary tumour 6 patient out of 35 (17.1\%) shown involvement of other site, the indication of surgery was to remove cord compression and restore neurological deficit. The most common site for metastasis was the dorsal spine for those of an unknown primary tumour 4 out of 5 patients $(80 \%)$, and for those of a known primary tumour it was the lumbar area 28 out of 35 patient $(80 \%)$. In conclusion metastatic spinal tumour of unknown primary was a common condition; it has similar demographic features, complications, and neurologic sequel with the spinal metastasis of known primary. Adenocarcinoma of the lung is the most common primary tumour proved after histopathology for spinal metastasis of unknown primary.

\title{
Keywords
}

\author{
Metastatic Spinal Tumour, Metastatic Spinal Tumour of Unknown Primary
}

\section{Introduction}

Spinal tumours are relatively rare disease; it affects about 10,000 Americans annually. It affects commonly young and middle-aged adults. More than 90 present of spine tumours are metastatic-cancer [1]. The spine is the third most common site for metastatic cancer, following lung and liver metastases [2]. Between 5\% and 40\% [3] of all cancer patients develop spinal metastases during the Course of their disease and approximately $70 \%$ of cancer patients have spinal metastatic disease at death [4]. The primary tumour was known in $50 \%$ to $80 \%$ of the spinal metastasis [5]. About $70 \%$ of metastatic spine tumours are in the thoracic spine, $20 \%$ in the lumbar region and $10 \%$ presented in the cervical spine [6] [7]. The single most important factor determining outcome is the level of neurologic function at initiation of therapy as well as the nature of the primary cancer, the number of lesions, the presence of distant non-skeletal metastases and the presence and/or severity of spinal-cord compression [6]-[8]. Common primary cancers that spread to the spine are lung, breast and prostate [8]. The time from diagnosis of the primary lesion to detection of the spinal metastasis was shown to be shortest in patients with lung cancer and longest in those with breast cancer. The highest 1-year survival rate was seen in metastases from breast cancer or prostate cancer whereas patients with metastases from lung or gastric cancer had the lowest 1-year survival rates [9].

The outlook for most cases of metastatic cancer to the spinal cord has historically been poor, outcomes are variable, based on a number of factors, including the type of cancer, the number of metastases to the spine and other areas of the body, the patients overall health, and degree of injury the metastasis has already inflicted on the spinal cord [10].

The aim of the study was to compare duration of symptoms, site of spinal involvement, other visceral or skeletal involvement, the neurological outcome, treatment outcome and complications between patients with unknown primary tumour with patients with known primary tumour.

\section{Patient and Methods}

We retrospectively interviewed 40 patients with spinal metastasis at neuroscience hospital in Baghdad between January 2010 and January 2014.

History taking and physical examination were done by a specialist neurosurgeon emphasising on neurological outcome and complications. Search for primary tumour and other visceral or skeletal metastasis by full blood picture, serum protein electrophoresis, C.T scans of chest, abdomen and pelvis, M.R.I of whole spine, X-ray of limbs and in some cases C.T scan of brain. Histopathological cell type of tumour was assessed by consultant pathologist.

Surgical decompression was done with or without stabilization as early as possible. Post-operatively further management was guided by the oncology team based on tissue histology from surgical specimens.

The study was approved and done under supervision of ethical and scientific committee of the neurosciences hospital.

Statistical analysis using SSPS version 19 was done using $\mathrm{T}$ test for assessment of $\mathrm{P}$ value to compare tow numerical results, $P$ value less than 0.05 was considered significant. 


\section{Results}

A total of 40 patient had surgery duo to metastatic spinal cord tumour 35 patient (87.5\%) had known primary lesion, while 5 patients (12.5\%) were of unknown primary/tumour even after full radiological investigations [see Table 1].

The median age at time of surgery was 64 years for the unknown primary with a range between 58 - 72 years, and for those of a known primary the median age was 61 year with a range between 45 - 70 years. Male are more common than female in both groups [see Table 1].

None of the 5 patient with secondary metastasis of an unknown primary lesion had other skeletal or visceral involvement [see Table 2].

There was no significant difference in 3 month duration of symptomsor in the neurological out come after surgery or the complication rate between the two groups [see Table 2].

The most common complication was deep wound infection, systemic, neurological, pulmonary and vascular [see Table 2].

Those 5 patients with the unknown primary tumour, the site of origin were diagnosed after surgical decompression and assessment of histopathology result. The 4 out of 5 with unknown primary was proved to be adenocarcinoma from the lung [see Table 3].

None of the 40 patients who had secondary spinal metastasis had cervical spine involvement. The dorsal spine was the common site for those of an unknown primary tumour $80 \%$. While the lumbar spine was the common site for those of a known primary tumour 28 out of 35 (80\%) [see Table 4].

The most common indication of surgery was neurological deficit in both groups as seen in Table 5.

Table 1. The demographic factors in the 2 groups of patients of metastatic spinal tumour of unknown primary and the group of known primary.

\begin{tabular}{lccc}
\hline \multicolumn{2}{c}{ Metastatic spinal tumour } & Unknown primary tumour & Known primary tumour \\
\hline \multicolumn{2}{c}{ Total no. of patients (40) } & $5(12.5 \%)$ & $35(87.5 \%)$ \\
\multicolumn{2}{c}{ Median age } & $64(58-72)$ years & $61(45-70)$ years \\
\cline { 3 - 4 } Gender & Male & $4 / 5(80 \%)$ & $22 / 35(62.8 \%)$ \\
& Female & $1 / 5(20 \%)$ & $13 / 35(37.2 \%)$ \\
\hline
\end{tabular}

Table 2. Differences between metastatic spinal tumour of unknown primary and a known primary, in relation to duration of symptoms, neurological outcome, complication rate, and presence of other skeletal or visceral involvement.

\begin{tabular}{|c|c|c|c|}
\hline Metastatic spinal tumour & Unknown primary tumour & Known primary tumour & $\mathrm{P}$ \\
\hline 3 month duration of symptoms & $4 / 5$ & $32 / 35$ & 0.4 \\
\hline Neurological out come after surgery & $4 / 5(80 \%)$ & 30/35 (85.7\%) & 0.7 \\
\hline Post-surgical complication rate & $25 \%$ & $28 \%$ & 0.6 \\
\hline Presence of other skeletal or visceral involvement & 0 & $6(17.15)$ & 0.03 \\
\hline
\end{tabular}

Table 3. Primary site of origin of metastatic spinal tumour of unknown primary and a known primary.

\begin{tabular}{crr}
\hline Metastatic spinal tumour & Unknown primary tumour & Known primary tumour \\
Lung & 4 patients (80\%) & 18 patients $(51.4 \%)$ \\
Colorectal & 1 patient (20\%) & 8 patient (22.8\%) \\
Breast & 7 patient (20\%) \\
Renal & 2 patient (5.71\%) \\
\hline
\end{tabular}


Table 4. The site of spine involvement comparison between metastatic spinal tumour of unknown primary and a known primary.

\begin{tabular}{ccc}
\hline Site of spine involved & Unknown primary tumour & Known primary tumour \\
\hline Cervical & 0 & 0 \\
Dorsal & 4 patients $(80 \%)$ & 7 patients $(20 \%)$ \\
Lumbar & 1 patient $(20 \%)$ & 28 patients $(80 \%)$ \\
Total & 5 patients & 35 patient \\
\hline
\end{tabular}

Table 5. Indication of surgery in both groups.

\begin{tabular}{ccr}
\hline Indication of surgery & Unknown primary tumour & Known primary tumour \\
\hline Pain & 1 patient (20\%) & 2 patient (5.7\%) \\
Neurological deficit & 3 patient (60\%) & 21 patients $(60 \%)$ \\
Pain \& neurological deficit & 1 patient (20\%) & 12 patients (34.2\%) \\
Total & 5 patients & 35 patients \\
\hline
\end{tabular}

\section{Discussion}

Malignant spinal cord compression is one of the most dreaded complications of cancer if untreated; it can lead to worsening neurologic function culminating in paralysis and sphincter incontinence. The most challenging aspect in the management of this complication is early diagnosis because the single most important factor determining outcome is the level of neurologic function at initiation of therapy [1].

Spinal metastasis from unknown primary tumour account 5 out of 40 patients (12.5\%) in our study, but other studies have reported this to be as $10 \%$ [2].

The present study is showing $80 \%$ more common male involvement than female in those with unknown primary tumour and in (62.8\%) in those with known primary tumour and this results are comparable with most other studies [3]-[5].

The mean age at time of presentation in both groups was nearly similar and consistent with other studies [3] [4], but salvatietal study was showing a younger age presentation with mean age was in the fourth decade [6].

Duration of symptoms prior to final diagnosis of spinal metastasis was nearly similar in both groups and comparable to other studies [3] [6].

The present study showed $60 \%$ of both patients groups had presented with neurological symptoms; this is contrasting with Enkaouaetal study [4] who reported in his series of 71 patients with spinal metastasis the main presenting symptoms was pain. While pain alone in our series was only $20 \%$ for those of unknown primary lesion and (5.7\%) for those with known primary tumour.

In the present study (80\%) of patient with unknown primary tumour and (85.7\%) of known primary tumour had the same or improved neurological function post operatively. The above result is lower than the Aizenbergetal study [5] who reported $94 \%$ of patients who had surgery of metastasis spinal cord tumours from unknown primary lesion had the same or improved neurological function post operatively. This result may suggest better surgical procedures and the post-operative rehabilitations offered for patients in Aizenbergetal study.

The complication rate in both groups of our study was comparable; $25 \%$ for those of unknown primary tumour and $28 \%$ for those with known primary tumours and similar to the rates reported in the literature [7].

None of our patient with unknown primary site had other skeletal or visceral involvement, while 6 patients (17.1\%) of a known primary tumour had other skeletal and visceral metastasis.

Visceral metastasis is described as a significant prognostic factor for survival in the studies of padalker [7], leithner [8] and van der linden [9].

Those patents with unknown primary tumour the site of origin was diagnosed after surgical removal and histopathology result shown (80\%) had adenocarcinoma from the lung and (20\%) had colorectal origin. Aizenberg et al. [5] found the most common primary site was the lung (31\%) this is supported by a review article by Hillen study [3] who reported that the commonest sites for unknown primary tumours were the lung (30\%) and pan- 
creas (20\%). While those with a known primary tumour the lung was (51.4\%) followed by colorectal (22.8\%), breast (20\%) and renal (5.7\%). Salvatietal study reported [6] in his study the pulmonary carcinoma in male and breast carcinoma in female as the most common site of primary tumour. Other study performed by Nottebaertstudy [10] shown that breast carcinoma was the most common primary tumour, occurring in $30 \%$ of his patients, followed by lung $17 \%$, prostate $10 \%$ and kidney $9 \%$.

The most common site of spinal metastasis in those with unknown primary site was the dorsal spine (80\%) followed by lumbar spine (20\%) similar to Douglas S. et al. [2] Study. while those group with known primary tumour in our study the most common site was lumbar spine $80 \%$ and $20 \%$ for the dorsal spine which was different from Salvatietal study [6] which was including 59 patients with dorsal spine involved was seen in 41 cases. Nottebaert et al. [10] study the thoracic segment was the most frequent site of cord compression.

\section{Conclusions}

In conclusion metastatic spinal tumour of unknown primary was a common condition; it carries a diagnostic challenge to the clinician; it forms $12.5 \%$ of the whole spinal secondaries; it has similar demographic features, complications, and neurologic sequel with the spinal metastasis of known primary. Adenocarcinoma of the lung is the most common primary tumour prove $\mathrm{d}$ after histopathology

This study shed a light on a common problem facing a treating neurosurgeon and raising the importance of early histopathology to detect the hidden primary which will result in early diagnosis and treatment.

\section{References}

[1] Yalamanchili, M. and Lesser, G.J. (2003) Malignant Spinal Cord Compression. Current Treatment Options in Oncology, 4, 509-516.

[2] Douglas, S., Huttenlocher, S., Bajrovic, A., Rudat, V., Schild, S.E. and Rades, D. (2012) Prognostic Factors for Different Outcomes in Patients with Metastatic Spinal Cord Compression from Cancer of Unknown Primary. BMC Cancer, 12, 261. http://dx.doi.org/10.1186/1471-2407-12-261

[3] Hillen, H.F.P. (2000) Unknown Primary Tumours. Postgraduate Medical Journal, 76, 690-693.

[4] Enkaoua, E.A., Doursounian, L., Chatellier, G., Mabesoone, F., Aim-ard, T. and Saillant, G. (1997) Vertebral Metastases-A Critical Appreciation of the Preoperative Prognostic Tokuhashi Score in a Series of 71 Cases. Spine, 22, 2293-2298. http://dx.doi.org/10.1097/00007632-199710010-00020

[5] Aizenberg, M.R., Fox, B.D., Suki, D., McCutcheon, I.E., Rao, G. and Rhines, L.D. (2012) Surgical Management of Unknown Primary Tumours Metastatic to the Spine. Journal of Neurosurgery: Spine, 16, 86-92. http://dx.doi.org/10.3171/2011.9.SPINE11422

[6] Salvati, M., Cervonil, L., Puzzilli, F., Raguso, M., Ciappetta, P. and Delfini, R. (1998) Metastatic Spinal Cord Compression. Clinical Remarks. Minerva Chirurgica, 53, 727-730.

[7] Podalkar, P. and Tow, B. (2011) Predicators of Survival in Surgically Treated Patients of Spinal Metastasis. Indian Journal of Orthopaedics, 45, 307-313. http://dx.doi.org/10.4103/0019-5413.82333

[8] Leither, A., Radl, R., Gruber, G., Hochegger, M., Leithner, K. and Welkerling, H. (2008) Predicative Value of Seven Preoperative Prognostic Scoring System for Metastasis. European Spine Journal, 17, 1488-1495. http://dx.doi.org/10.1007/s00586-008-0763-1

[9] Vander Linden, Y.M., Dijkstra, Sp, Vonk, E.J., Marijnen, C.A. and Leer, J.W. (2005) Predication of Survival in Patients with Metastases in the Spinal Column; Results Based on a Randomized Trial of Radio Therapy. Cancer, 103, 320-328.

[10] Nottebaert, M., Von Hochstetter, A.R., Exner, G.U. and Schreiber, A. (1987) Metastatic Carcinoma of the Spine. A Study of 92 Cases. Orthop, 11, 345-348. 